\title{
Chlamydophila abortus em animais de produção
}

\author{
Chlamydophila abortus in production animals
}

Francielle Gibson da Silva ${ }^{1}$ Julio Cesar de Freitas $^{2^{*}}$ Ernst Eckehardt Müller $^{2}$

- REVISÃO BIBLIOGRÁFICA -

\section{RESUMO}

A Chlamydophila abortus (anteriormente classificada como Chlamydia psittaci sorotipo 1) tem sido descrita em muitos países, associada principalmente com distúrbios reprodutivos em ovinos, bovinos e caprinos. $O$ aborto enzoótico dos ovinos e caprinos e o aborto epizoótico dos bovinos são as doenças mais importantes causadas por esta bactéria. No Brasil, as pesquisas com $\boldsymbol{C}$. abortus são praticamente inexistentes. O objetivo desta revisão é apresentar informações sobre modificações taxonômicas, ciclo de vida, epidemiologia, patogenia, sinais clínicos e diagnóstico da infecção por C. abortus principalmente em ovinos, bovinos $e$ caprinos.

Palavras- chave: Chlamydophila abortus, ovino, bovino, caprino, aborto, problema reprodutivo.

\section{ABSTRACT}

Chlamydophila abortus (previously known as Chlamydia psittaci serovar 1) has been reported in many countries, associated with reproductive disorders in sheep, cattle, and goats. The enzootic abortion of sheep and goats and the epizootic bovine abortion are the most important diseases produced by this bacterium. In Brazil, there is scarce information about $\boldsymbol{C}$. abortus. The objective of this review is to show information about taxonomic changes, life cycle, epidemiology, pathogenesis, clinical signs and diagnosis of $\boldsymbol{C}$. abortus in sheep, cattle and goats.

Key words: Chlamydophila abortus, sheep, cattle, goat, abortion, reproductive disorders.

\section{INTRODUÇÃO}

As bactérias da família Chlamydiaceae são Gram negativas possuem forma cocóide, são intracelulares obrigatórias, com ciclo de vida que se alterna entre formas infectantes (corpos elementaresCE) e vegetativas (corpos reticulados-CR). Estas bactérias infectam várias espécies de animais domésticos e selvagens, além do homem, causando manifestações clínicas bastante variadas (SCHACHTER \& CALDWELL, 1980; CORSARO et al., 2003). Dependendo da espécie animal envolvida, pode ocorrer aborto, pneumonia, rinite, conjuntivite, artrite e enterite, entretanto é comum a ocorrência da infecção em animais clinicamente sadios (SHEWEN, 1980; EVERETT, 2000).

Pelas suas características, as bactérias da ordem Chlamydiales possuem apenas uma família, a Chlamydiaceae. Até recentemente era reconhecido apenas o gênero Chlamydia, com duas espécies, a trachomatis e a psittaci, com vários sorovares (EVERETT, 2000). Com o desenvolvimento de técnicas de biologia molecular, principalmente análises do gene $16 \mathrm{~S}$ e $23 \mathrm{~S}$ do RNAr, foi possível a divisão da família Chlamydiaceae em dois gêneros: Chlamydia e Chlamydophila e em nove espécies: Chlamydia trachomatis, C. suis, C. muridarum, Chlamydophila psittaci, C. pneumoniae, C. pecorum, C. felis, $C$. caviae e $\boldsymbol{C}$. abortus (PUDJIATMOKO et al., 1997;

${ }^{1}$ Curso de Pós-graduação em Ciência Animal, Centro de Ciências Agrárias (CCA), Universidade Estadual de Londrina (UEL), Londrina, Paraná, Brasil.

²Departamento de Medicina Veterinária Preventiva, CCA, UEL, Londrina, Paraná, Brasil.

*Autor para correspondência: Prof. Dr. Julio Cesar de Freitas, Rodovia Celso Garcia Cid (PR 445), Km 380, CP 6001, 86051990, Londrina, Paraná, Brasil. Tel: (43) 3371-4765; Fax: (43) 3371-4714, E-mail: freitasj@uel.br. 
TAKAHASHI et al., 1997; EVERETT, 2000; HERRMANN et al., 2000).

A nova espécie Chlamydophila abortus (C. abortus), anteriormente classificada como Chlamydia psittaci $(\boldsymbol{C}$. psittaci) sorovar 1, é freqüentemente associada a distúrbios reprodutivos em ovinos, caprinos, bovinos e esporadicamente em suínos e eqüinos (EVERETT, 2000). O aborto enzoótico dos ovinos e caprinos e o aborto epizoótico dos bovinos são as manifestações mais importantes e têm sido documentados por vários autores (NANDA et al., 1992; GRIFFITHS et al., 1995; OGINO et al., 1996; RODOLAKIS et al., 1998; REKIKI et al., 2002; SZEREDI $\&$ BACSADI, 2002). O aborto enzoótico dos ovinos foi primeiramente diagnosticado por STAMP et al. (1950) na Escócia e a partir de 1956, em vários países da Europa, enquanto STORZ et al. (1960) realizaram o primeiro isolamento de bactérias da família Chlamydiaceae a partir de aborto bovino.

Relatos em vários países mostram a atual importância da $\boldsymbol{C}$. abortus nos problemas reprodutivos de ovinos e bovinos (WITTENBRINK et al., 1993; DANNATT et al., 1998; REKIKI et al., 2002; AMIM, 2003; LONGBOTTOM \& COULTER, 2003). Na Suíça, a prevalência do aborto enzoótico dos ovinos foi estimada em aproximadamente 19\% (BOREL et al., 2004) e na Espanha, a soroprevalência foi de 50,5\% nos 52 rebanhos estudados (MAINAR-JAIME et al., 1998). Levantamentos epidemiológicos realizados na Índia por NANDA et al. (1992) detectaram 80,7\% de bovinos sororreatores para $\boldsymbol{C}$. abortus, enquanto na Itália PUGLIESE et al. (1991) encontraram 53,7\% bovinos positivos utilizando a reação de fixação de complemento.

No Brasil, não há dados sobre a ocorrência de infecção por $\boldsymbol{C}$. abortus em ovinos e caprinos. Na espécie bovina, os dados também são praticamente inexistentes, o que dificulta o conhecimento da doença no país. O primeiro trabalho relatando a ocorrência de bactérias da família Chlamydiacae no país foi realizado em bubalinos do Estado do Pará por FREITAS \& MACHADO (1988), que isolaram $\boldsymbol{C}$. psittaci de animais abatidos para consumo, com quadro de serosite. A seguir, no Rio de Janeiro, ROMIJN \& LIBERAL (1990) também isolaram $\boldsymbol{C}$. psittaci de pulmões de bezerros com pneumonia. GOMES et al. (2001), no Rio Grande do Sul, isolaram $\boldsymbol{C}$. psittaci a partir de amostras de sêmen de touros com vesiculite seminal, sendo que os animais também apresentaram títulos positivos na fixação de complemento. SOUZA (2002), utilizando fêmeas bovinas com distúrbios reprodutivos do estado de São Paulo, detectou $12,5 \%$ de animais positivos na reação de fixação de complemento no único levantamento sorológico para o gênero Chlamydophila do país.

Apesar da existência de diferentes técnicas de diagnóstico, no Brasil são poucos os laboratórios veterinários de doenças infecciosas que se dedicam à C. abortus, dificultando o desenvolvimento de estudos epidemiológicos direcionados para o conhecimento da verdadeira situação da doença no país. Assim, esta revisão de literatura tem o objetivo de apresentar informações sobre modificações taxonômicas, ciclo de vida, epidemiologia, patogenia, principais sinais clínicos e diagnóstico da infecção por $\boldsymbol{C}$. abortus principalmente em ovinos, bovinos e caprinos.

\section{Ciclo de vida}

Bactérias da família Chlamydiaceae possuem um ciclo de vida único, porém dimórfico. $\mathrm{O}$ ciclo de replicação ocorre no citoplasma da célula hospedeira infectada e se inicia pela adesão do CE na membrana da célula, seguido por internalização que dá origem a um vacúolo endossomal (VANROMPAY et al., 1995). Um mecanismo ainda desconhecido, impede a fusão de lisossomos primários permitindo a sobrevivência do CE, no interior do endossomo, que com o início da divisão por fissão binária, se diferencia em CR. Após a replicação, estes CR são reorganizados, através de formas intermediárias, em novos CE. Aproximadamente 48 a $72 \mathrm{~h}$ após infecção in vitro, uma inclusão clamidial pode conter centenas de estruturas em vários estágios de desenvolvimento, incluindo $\mathrm{CE}$, CR e formas intermediárias (VANROMPAY et al., 1995; EVERETT, 2000). A liberação dos CE pode ocorrer por lise da célula hospedeira ou por liberação de inclusões clamidiais pela membrana celular, entretanto estes mecanismos não estão bem esclarecidos (VANROMPAY et al., 1995).

\section{Epidemiologia}

O aborto enzoótico ovino tem ampla distribuição em regiões criadoras de ovinos (O.I.E., 2005). AITKEN et al. (1990) e LEONARD et al. (1993) consideraram a C. abortus a maior causa de abortamento em ovelhas na Europa, sendo responsável por $45 \%$ dos abortamentos diagnosticados no Reino Unido, onde aproximadamente 1,5 milhão de ovelhas (8,6 \% dos rebanhos) são infectadas anualmente. Entretanto, em áreas tradicionais da ovicultura como Austrália e Nova Zelândia, esta enfermidade não é considerada um problema (LONGBOTTOM \& COULTER, 2003). O aborto enzoótico caprino é semelhante em severidade ao dos ovinos, porém faltam dados epidemiológicos, não apenas no Brasil, que permitam a compreensão da disseminação e do impacto 
econômico decorrente da doença (AITKEN et al., 1990; LONGBOTTOM\& COULTER, 2003).

A ocorrência de surtos de abortamentos em rebanhos leiteiros da Inglaterra despertou o interesse pela pesquisa de $\boldsymbol{C}$. abortus em distúrbios reprodutivos (DANIEL et al., 1993). A doença é descrita como aborto epizoótico bovino por suas características epidemiológicas e está disseminada em quase todos os países da Europa e nos Estados Unidos da América (STORZ et al., 1968; SHEWEN, 1980; HOLLIMAN et al., 1994). O conhecimento da ocorrência da doença em um número restrito de países, provavelmente é resultante da falta de pesquisas com $\boldsymbol{C}$. abortus.

Abortamentos em suínos e eqüinos por $\boldsymbol{C}$. abortus têm sido relatados, entretanto acredita-se que ocorram em menor freqüência quando comparado às espécies ovina e bovina (THOMA et al., 1997; LONGBOTTOM \& COULTER, 2003).

Os animais podem se infectar $\operatorname{com}$ C. abortus em qualquer idade ou estação do ano (LONGBOTTOM \& COULTER, 2003; DeGRAVES et al., 2004). As fontes de infecção mais comuns são os fetos abortados, a placenta e as descargas uterinas. Após o abortamento ou parto, $\boldsymbol{C}$. abortus são eliminadas em grande número nos fluidos, nos envoltórios fetais e na placenta e continuam sendo eliminadas até 7-14 dias nas secreções vaginais (LONGBOTTOM \& COULTER, 2003). A transmissão pode ser vertical, por infecção congênita do feto, horizontal e por contaminação direta por via alimentar, conjuntival ou genital. A infecção por ingestão de CE é a via de transmissão mais importante (DeGRAVES et al., 2004)

Poucas são as evidências de transmissão sexual de $\boldsymbol{C}$. abortus em ovinos, entretanto em carneiros com infecções crônicas a $\boldsymbol{C}$. abortus foi isolada do sêmen e glândulas sexuais acessórias (RODOLAKIS; BERNARD, 1977). A ocorrência de vesiculites, epididimites e orquites podem resultar na eliminação de $\boldsymbol{C}$. abortus pelo sêmen, causando infertilidade, diretamente pelo desenvolvimento de infecção intrauterina pós-monta ou inseminação artificial ou indiretamente, pela pioespermia que leva à diminuição da qualidade do sêmen (STORZ et al., 1968; LONGBOTTOM \& COULTER, 2003). Em bovinos, trabalhos experimentais demonstraram a participação da via sexual na epidemiologia da doença, resultando em redução dos índices de fertilidade (BOWEN et al., 1978; PAPP; SHEWEN, 1996; JONES, 1999). DeGRAVES et al. (2004) demonstraram que novilhas que receberam C. abortus diretamente no útero apresentaram um risco 8,5 vezes maior de desenvolver infertilidade quando comparado às fêmeas que não foram expostas à bactéria.
Patogenia

A maioria dos estudos de infecção natural ou experimental por $\boldsymbol{C}$. abortus foi realizada utilizando a espécie ovina como modelo, assim dados sobre a epidemiologia e patogenia da infecção nesta espécie animal são extrapolados para as demais espécies acometidas (LONGBOTTOM \& COULTER, 2003).

Estudos realizados com ovelhas indicam as tonsilas como primeiro local de replicação, quando a infecção ocorre pela ingestão de CE, ocorrendo posteriormente a disseminação pelo sangue, linfa e outros órgãos (JONES \& ANDERSON, 1988). Nos animais não gestantes a infecção torna-se latente e as C. abortus permanecem possivelmente em tecido linfóide, por um processo mediado por citocinas (ENTRICAN et al., 1998). Nesta fase de latência, a $\boldsymbol{C}$. abortus não é detectada por métodos laboratoriais utilizados atualmente e somente na gestação subseqüente a bactéria começa a se replicar novamente, provavelmente por mecanismos imunomediados. Este início de bacteremia é relacionado com o desenvolvimento da placenta. NAVARRO et al., (2004) relataram que o início do desenvolvimento de hematomas na interface materno-fetal no hilo dos placentomas ocorre aproximadamente no $60^{\circ}$ dia de gestação, entretanto nenhuma alteração patológica ocorre até o $90^{\circ}$ dia de gestação. A C. abortus invade as células do epitélio coriônico de vários placentomas, onde se replica, formando inclusões intracitoplasmáticas. A infecção se dissemina para regiões intercotiledonárias do córion, causando lesão epitelial, edema e inflamação. Estas alterações resultam em coloração avermelhada e espessamento das membranas da placenta, característico da doença (ENTRICAN etal., 1998; LONGBOTTOM \& COULTER, 2003; NAVARRO et al., 2004).

Os mecanismos responsáveis pelo abortamento não são claros, mas acredita-se ser resultado da associação das lesões epitélio coriônicas, que levam ao comprometimento da troca de oxigênio e de nutrientes entre mãe e feto, e das alterações patológicas fetais, principalmente necrose focal de fígado e com menor frequiência, nos pulmões, baço, cérebro e linfonodos (BUXTON et al., 1990; 2002). Infecções por $\boldsymbol{C}$. abortus estimulam uma forte resposta inflamatória no animal, principalmente pela ativação de interferons (IFN- $\gamma$ ) e fator de necrose tumoral (TNF- $\alpha$ ), que pode tanto controlar o processo infeccioso como agravar as alterações patológicas (KEER et al., 2005). Alterações nas concentrações plasmáticas dos hormônios progesterona e $17 \beta$ estradiol e de prostaglandina $\mathrm{E}$ no líquido aminiótico e alantóideo, são conseqüênciàs da infecção placentária pela $C$

Ciência Rural, v.36, n.1, jan -fev, 2006. 
abortus que também podem resultar em parto prematuro (KEER et al., 2005).

Os animais infectados congenitamente que não são abortados, geralmente nascem fracos, e quando sobrevivem, freqüentemente apresentam infecção latente que se manifesta logo na primeira gestação (PAPP \& SHEWEN, 1996). O processo de saída de latência e desenvolvimento da doença é igual ao de um animal infectado por transmissão horizontal.

Sinais clínicos

Em ovinos, bovinos e caprinos, geralmente a ocorrência de abortamentos nas últimas duas ou três semanas de gestação é o primeiro indício de que a infecção por $\boldsymbol{C}$. abortus está instalada no rebanho (LONGBOTTOM \& COULTER, 2003; NAVARRO et al., 2004). Após a instalação da infecção no rebanho, os índices de abortamento são superiores a $30 \%$ no primeiro ano, permanecendo entre $5-10 \%$ nos anos seguintes se não forem adotadas medidas de controle (LONGBOTTOM; COULTER, 2003). Com o desenvolvimento de imunidade específica contra $\boldsymbol{C}$. abortus, dificilmente um animal infectado aborta duas vezes, entretanto, alguns animais podem desenvolver imunidade incompleta e abortarem novamente, elevando o percentual de abortamentos no rebanho no segundo ano de infecção (PAPP et al., 1994). A eliminação intermitente de $\boldsymbol{C}$. abortus nestes animais pode ocorrer por até três anos (KEER et al., 2005).

Os fetos abortados podem apresentar-se normais ou com graus variados de edema. A placenta encontra-se espessada, com coloração vermelhoamarelada e exudato vaginal de coloração rósea suja pode ser observado até 7 a 10 dias pós-abortamento (LONGBOTTOM \&; COULTER, 2003). Em bovinos e caprinos, a ocorrência de retenção placentária, endometrites e vaginites são freqüentes e ocasionalmente pode ocorrer metrite e morte por infecção bacteriana secundária (RODOLAKIS et al., 1984; WITTENBRINK et al., 1993a,b; PAPP et al, 1994). Além do abortamento, outros sinais de distúrbios reprodutivos como nascimento prematuro e de animais fracos e natimortos também são associados à infecção por $\boldsymbol{C}$. abortus. Animais recém-nascidos podem apresentar quadros respiratórios inespecíficos e de encefalites, que geralmente morrem 48 horas após o nascimento, mesmo recebendo tratamento de suporte (LONGBOTTOM \& COULTER, 2003).

Especificamente em bovinos, outros sinais clínicos como repetição de cio em intervalos irregulares, aumento no intervalo de partos e de número de serviços ou de inseminação é observado nos rebanhos infectados (SHEWEN, 1980; WITTENBRINK et al., 1993b).
Nos machos em idade reprodutiva, os sinais clínicos observados são vesiculites, epididimites e orquites (STORZ et al., 1968, GOMES et al., 2001).

\section{Diagnóstico}

A C. abortus pode ser detectada diretamente na placenta e órgãos fetais, na excreção vaginal até 14 dias pós-abortamento ou no sêmen, pelo cultivo em ovo embrionado ou cultivo de células, imunofluorescência direta, imunohistoquímica, ELISA e testes de detecção de DNA (STORZ et al., 1968; JOHNSON et al., 1983; MOORE et al., 1987; BROWN \& NEWMAN, 1989; DAGNAL \& WILSMORE, 1990; GRIFFITHS et al., 1992; BUXTON et al., 1996; LAROUCAU et al., 2001).

A amplificação do DNA pela reação em cadeia da polimerase (PCR) tem sido indicada para a identificação das diferentes espécies pertencentes à família Chlamydiaceae (EVERETT, 2000; MADICO et al., 2000). Atualmente, outras técnicas como PCR em tempo real, Multiplex e sistemas fluorescentes de detecção da seqüência amplificada têm sido utilizadas (CREELAN; McCULLOUGH, 2000; MADICO et al., 2000). A diferenciação da $\boldsymbol{C}$. abortus pode ser realizada utilizando oligonucleotídios iniciadores (primers) específicos, análise do polimorfismo no comprimento de fragmentos de restrição e também pelo seqüenciamento genômico dos produtos obtidos na PCR (WATSON et al., 1991; SHEEHY et al., 1996; EVERETT, 2000; HARTLEY et al., 2001).

Para a detecção de anticorpos contra $\boldsymbol{C}$. abortus diferentes testes como ELISA, imunofluorescência indireta e fixação de complemento têm sido utilizados (OIE, 2004). Apesar de apresentarem vários inconvenientes como a dificuldade de padronização e produção de antígenos, estes testes apresentam alta sensibilidade e facilidade de execução em todo o rebanho, por isso são extensivamente empregadas principalmente nos países onde existem programas de controle da doença (DONN et al., 1997; KALTENBOECK et al., 1997; GOMES et al., 2001). A técnica de imunofluorescência indireta é amplamente utilizada na rotina de diagnóstico das infecções por outras espécies de Chlamydia e Chlamydophila em humanos, porém não tem sido usada na medicina veterinária (PEREZ-MARTINEZ et al., 1986; MARKEY et al., 1993; GRIFFTHS et al., 1995; BUENDÍA et al., 2001; OIE., 2004). Entre os diferentes testes para a detecção de anticorpos, o de fixação de complemento é o mais utilizado no diagnóstico de rotina, sendo o teste recomendado pelo Office International dês Epizooties (OIE). Ele detecta anticorpos produzidos contra o epítopo do antígeno lipopolissacarídeo 
presente em todas as espécies da família Chlamydiaceae (EVERETT, 2000).

\section{Tratamento e controle}

A família Chlamydiaceae é suscetível a vários antibióticos, sendo as tetraciclinas os antibióticos preferenciais para o tratamento das infecções. A oxitetraciclina de longa ação $\left(20 \mathrm{mg} \mathrm{kg}^{-1}\right.$, intramuscular) é a mais usada nas infecções por $\boldsymbol{C}$. abortus nos animais de produção (LONGBOTTOM \& COULTER, 2003). A administração de antibióticos, durante um surto, não elimina a infecção do rebanho, mas suprime a replicação do microrganismo, diminuindo a severidade dos sinais clínicos e aumentando o número de nascimentos de animais vivos. Para melhores resultados, o tratamento de ser iniciado após o $90^{\circ}$ dia de gestação, quando são detectadas as primeiras alterações na placenta e uma segunda dose deve ser administrada duas semanas após a primeira dose (BUXTON, 1990; LONGBOTTOM \& COULTER, 2003). A antibioticoterapia deve ser sempre associada ao correto manejo sanitário e à profilaxia com vacinas. Estas medidas são mais eficientes tanto para impedir a entrada da $\boldsymbol{C}$. abortus como para o controle da infecção (LONGBOTTOM \& COULTER, 2003).

Em rebanhos considerados livres de $\boldsymbol{C}$. abortus, os animais de reposição também devem ser procedentes de áreas livres, monitoradas sorologicamente (ENTRICAN et al., 2001). Quando a infecção já se instalou na propriedade, o manejo sanitário inclui o isolamento, por pelo menos duas a três semanas, de todas as fêmeas que abortaram, destruição das placentas e limpeza dos piquetes contaminados durante os abortamentos, além da vacinação (LONGBOTTOM \& COULTER, 2003).

Atualmente existem dois tipos de vacinas disponíveis comercialmente apenas para ovinos, uma inativada e outra, viva atenuada, entretanto nenhuma confere proteção absoluta (GARCIA DE LA FUENTE et al. 2004; OIE., 2004). As vacinas inativadas são preparadas no saco da gema de ovos embrionados ou em cultivos celulares, utilizando apenas frações ou todo o microrganismo e adjuvantes oleosos. Este tipo de vacina pode ser administrado em animais gestantes e o período de cobertura pode chegar até dois anos (O.I.E., 2004). A vacina viva atenuada utiliza uma estirpe mutante termolábil, quimicamente induzida, de $\boldsymbol{C}$. abortus, por isso, somente animais não gestantes podem ser vacinados. Esta vacina confere imunidade por até três gestações e reduz drasticamente a eliminação de $\boldsymbol{C}$. abortus no ambiente e o número de abortamentos, importantes para a erradicação da doença (GARCIA DE LA FUENTE et al. 2004; OIE., 2004;
LONGBOTTOM \& LIVINGSTONE, 2004). Os dois tipos de vacina podem ser administrados via intramuscular ou subcutânea e a vacinação deve ser sempre antes da monta natural ou inseminação artificial (GARCIA DE LA FUENTE et al. 2004; OIE., 2004).

\section{CONCLUSÃO}

Apesar da C. abortus ser identificada com freqüência em vários países, principalmente nos abortamentos de ovinos e caprinos (aborto enzoótico) e de bovinos (aborto epizoótico), no Brasil não há dados epidemiológicos suficientes que demonstrem a participação desta bactéria nos problemas reprodutivos das espécies de animais de produção. Espera-se que as informações, apresentadas nesta revisão, tenham demonstrado a necessidade de desenvolver estudos sobre o envolvimento deste microrganismo nos problemas reprodutivos em nosso país, visando principalmente medidas que permitam a prevenção da infecção por esta bactéria nas espécies de animais de produção.

\section{REFERÊNCIAS}

AITKEN, I.D. et al. Enzootic abortion of ewes. Veterinary Record, v.126, p.136-138, 1990.

AMIM, A.S. Comparison of polymerase chain reaction and cell culture for the detection of Chlamydophila species in the semen of bulls, buffalo-bulls and rams. Veterinary Journal, v.166, p.86-92, 2003.

BOREL, N. et al. Seroprevalences for ovine enzootic abortion in Switzerland. Preventive Veterinary Medicine, v.65, p.205-216, 2004.

BOWEN, R.A. et al. Mechanisms of infertility in genital tract infections due to Chlamydia psittaci transmitted through contamined semen. Journal of Infectious Diseases, v.138, p.95-98, 1978

BROWN, P.A.; NEWMAN, J.A. Methods of chlamydial antigen detection. Journal American Veterinary Medical Association, v.195, n.11, p.1567-1570, 1989.

BUENDÍA, A.J. et al. Field evaluation of a new commercially avaiable ELISA base on a recombinat antigen for diagnosing Chlamydophila abortus (Chlamydia psittaci serotype 1) infection. Veterinary Microbiology, v.78, p.229-239, 2001.

BUXTON, D. et al. Observations on the pathogenesis of Chlamydia psittaci infection of pregnant sheep. Journal of Comparative Pathology, v.102, p.221-237, 1990.

BUXTON, D. et al. Pathogenesis of Chlamydia psittaci infection in sheep: detection of the organism in a serial study of the lymph node. Journal of Comparative Pathology, v.114, p.221-230, 1996. 
BUXTON, D. et al. Ovine chlamydial abortion; histopathological identification and analysis of immune cells in placental tissues. Journal of Comparative Pathology, v.127, p.133-141, 2002

CORSARO, D. et al. Increasing diversity within Chlamydiae. Critical Reviews in Microbiology, v.29, n.1, p.37-78, 2003.

CREELAN, J. L.; McCULLOUGH, S.J. Evaluation of strainspecifc primer sequences from an abortifacient strain of Chlamydophila abortus (Chlamydia psittaci) for the detection of EAE by PCR. FEMS Microbiology Letters, v.190, p.103$108,2000$.

DAGNAL, G.J.R.; WILSMORE, A.J. A simple staining method for the identification of chlamydial elementary bodies in the fetal membranes of sheep affected by the Ovine Enzootic Abortion. Veterinary Microbiology, n.21, p.233-239, 1990.

DANIEL, R.G. et al. Bovine chlamydiosis in the United Kingdom. Veterinary Record, v.133, p.352-355, 1993.

DANNATT, L. et al. Investigation of a possible role for chlamydia in a new disease syndrome in dairy cattle. Veterinary Record, v.19, n.26, p.691-693, 1998.

DeGRAVES, F.J. et al. Reinfection with Chlamydophila abortus by uterine and indirect cohort routes reduces fertility in cattle preexposed to Chlamydophila. Infection and Immunity, v.72, n.5, p.2538-2545, 2004

DONN, A. et al. Standardizzazione della tecnica di fissazione del complemento per la dimostrazione di anticorpi antiChlamydia nel siero di sangue. II Progresso Veterinario, v.4 p.125-128, 1997

ENTRICAN, G. et al. Cytokines and the protective host immune response to Chlamydia psittaci. Comparative Immunology, Microbiology and Infectious Diseases, v. 21 , p.15-26, 1998

ENTRICAN, G. et al. Chlamydial infection in sheep: immune control versus fetal pathology. Journalof the Royal Society of Medicine, v.94, p.273-277, 2001

EVERETT, K. D.E. Chlamydia and Chlamydiales: more than meets the eye. Veterinary Microbiology, v.75, p.109-126, 2000.

FREITAS, J.A.; MACHADO, R.D. Isolamento de Chlamydia psittaci em búfalos abatidos para consumo em Belém, Pará, Brasil. Pesquisa Veterinária Brasileira, v.8, p.43-50, 1988

GARCIA DE LA FUENTE et al. Efficacy of different comercial and new inactivated bacines against ovine enzootic abortion. Veterinary Microbiology, v.100, p.65-76, 2004.

GOMES, M.J.P. et al. Isolamento de Chlamydia psittaci em touros com vesiculite seminal, no Rio Grande Do Sul. A Hora Veterinária, n.119, p.43-46, 2001

GRIFFITHS, P.C. et al. Antigenic and morphological differentiation of placental and intestinal isolates of Chlamydia psittaci of ovine origin. Veterinary Microbiology, v.30, p.165-177, 1992.

GRIFFITHS, P.C. et al. Epizootic bovine abortion in a dairy herd: characterization of a Chlamydia psittaci isolate and antibody response. British Veterinary Journal, v.151, n.6, p.683-693, 1995.

HARTLEY, J.C. et al. PCR detection and molecular identification of Chlamydiaceae species. Journal of Clinical Microbiology, v.39, n.9, p.3072-3079, 2001.

HERRMANN, B. et al. Characterization of the rnp B gene and the RNase P RNA in the order Chlamydiales. International Journal of Systematic Evolutionary Microbiology, v.50, p.149-158, 2000.

HOLLIMAN, A. et al. Chlamydiosis and abortion in a dairy herd. Veterinary Record, v.134, p.500-502, 1994.

JOHNSON, F.W. A. et al. Direct isolation of the agent of enzootic abortion of ewes (Chlamydia psittaci) in cell cultures. Veterinary Record, v.113, p.413-414, 1983.

JONES, G.E. Chlamydial diseases of the reproductive tract of domestic ruminants. In: HITCHCOCK, P.J. et al. Sexually transmitted diseases and adverse outcomes of pregnancy. Washington: ASM, 1999. p.293-309.

JONES, G.E.; ANDERSON, I.E. Chlamydia psittaci: is tonsilar tissue the portal of entry in ovine enzootic abortion? Research in Veterinary Medicine, v.44, p.260-261, 1988

KALTENBOECK, B. et al. Use of syntethic antigens improves detection by enzyme-linked immunosorbent assay of antibodies against abortigenic Chlamydia psittaci in ruminants. Journal of Clinical Microbiology, v.35, p.2293-2298, 1997.

KEER, K. et al. Immunopathology of Chlamydophila abortus infection in sheep and mice. Research in Veterinary Science, v.78, p.1-7, 2005

LAROUCAU, K. et al. Improved sensitivity of PCR for Chlamydophila using pmp genes. Veterinary Microbiology, v.82, p.155-164, 2001

LEONARD, C. et al. An estimate of the prevalence of enzootic abortion of ewes in Scotland. Veterinry Record, v.133, p.180183,1993

LONGBOTTOM, D.; COULTER, L.J. Animal chlamydioses and zoonotic implications. Journal of Comparative Pathology, v.128, p.217-244, 2003.

LONGBOTTOM, D.; LIVINGSTONE, M. Vaccination against chlamydial infections of man and animals. Veterinary Journal, (in press). Acessado em: 05/04/2005. Online disponível em: www.sciencedirect.com/science.

MADICO, G. et al. Touchdown enzyme time release- PCR for detection and identification of Chlamydia trachomatis, Chlamydia pneumoniae and Chlamydia psittaci: using the $16 \mathrm{~S}$ 23S spacer rRNA genes. Journal of Clinical Microbiology, v.38, p.1085-1093, 2000.

MAINAR-JAIME, R.C. et al. Epidemiologic study of chlamydial infection in sheep farms in Madrid, Spain. Small Ruminant Research, v.28, p.131-138, 1998.

MARKEY, B.K. et al. Comparison of serological tests for diagnosis of Chlamydia psittaci infection of sheep. Veterinary Microbiology, v.36, p.233-252, 1993 
MOORE, F.M. et al. Detection of chlamydial inclusions in cell cultures or biopsy tissue by alkaline phosphatase anti-alkaline phosphatase staining. Journal of Clinical Microbiology, v. 25 , p.1864-1867, 1987.

NANDA, N.K. et al. Diagnosis of bovine chlamydial abortion in cattle. Indian Veterinary Journal, v.69, p.483-486, 1992.

NAVARRO, J.A. et al. Kinetics of infection and effects on the placenta of Chlamydophila abortus in experimentally infected pregnant ewes. Veterinary Pathology, v.41, p.498-505, 2004.

OIE (OFFICE INTERNATIONAL DES ÉPIZOOTIES). Ovine chlamydiosis. In: Manual of standards for diagnostic tests and vaccines. 4.ed. Paris: OIE, 2000. Acessado em: 22/03/ 2004. Online. Disponível em http://www.oie.int/.

OIE. (OFFICE INTERNATIONAL DES ÉPIZOOTIES). 2005. Acessado em: 25/07/2005. Online. Disponível em http:// www.oie.int/.

OGINO, H. et al. Pathology of bovine abortion and newborn calf death caused by dual infection with Chlamydia psittaci and infectious bovine rhinotracheitis virus. Journal of Veterinary Medicine Science, v.58, n.1, p.67-70, 1996.

PAPP, J.R. et al. Abortion and subsequent excretion of chlamydiae from the reproductive tract of sheep during estrus. Infection and Immunity, v.62, p.3786-3792, 1994.

PAPP, J.R.; SHEWEN, P.E. Localization of chronic Chlamydia psittaci infection in the reproductive tract of sheep. Journal of Infectious Diseases, v.174, p.1296-1302, 1996.

PEREZ-MARTINEZ, J.A.; STORZ, J. Bovine chlamydial abortion: serodiagnosis by modified complement-fixation and indirect inclusion fluorescence tests and enzyme-linked immunosorbent assay. American Journal of Veterinary Research, v.47, p.1501-1506, 1986.

PUDJIATMOKO, F.H. et al. Phylogenetic analysis of the genus Chlamydia based on $16 \mathrm{~S}$ rRNA gene sequences. International Journal of Systematic Bacteriology, v.47, p.425-431, 1997.

PUGLIESE, A. et al. Epidemiological studies on the prevalence of bovine chlamydiosis in Calabria. Atti dela Societa Italiana di Buiatria, v.23, p.183-188, 1991.

REKIKI, A. et al. Isolation and characterisation of local strains of Chlamydophila abortus (Chlamydia psittaci serotype 1) from Tunisia. Veterinary Research, v.33, p.215-222, 2002.

RODOLAKIS, A.; BERNARD, K. Isolament de chlamydia des organes genitaux de beliers atteints d'epididymite. Bulletin de l'Academie Veterinaire de France, v.50, p.65-70, 1977.

RODOLAKIS, A. et al. Chlamydia psittaci experimental abortion in goats. American Journal of Veterinary Research, v.45, p.2086-2089, 1984.

RODOLAKIS, A. et al. Recent advances on ovine chlamydial abortion. Veterinary Research, v.29, p.275-288, 1998.
ROMIJN, P.C.; LIBERAL, M.H.T. Cultivo de Chlamydia em diferentes sistemas celulares: um estudo comparativo. Pesquisa Agropecuária Brasileira, v.25, n.1, p.15-18, 1990.

SCHACHTER, J.; CALDWELL, H.D. Chlamydiae. Annual Review of Microbiology, v.34, p.285-309, 1980.

SHEEHY, N. et al. Diferentiation of Chlamydia psittaci and C. pecorum strains by species-specific PCR. Journal of Clinical Microbiology, v.34, n.12, p.3175-3179, 1996.

SHEWEN, P.E. Chlamydial infection in animals: a review. Canadian Veterinary Journal, v.21, p.2-11, 1980.

SOUZA, C.A. I. Ocorrência de anticorpos anti- Chlamydophila em bovinos e sua relação com distúrbios reprodutivos. 2002. 63f. Dissertação (Mestrado em Epidemiologia Aplicada às Zoonoses e Saúde Animal) - Faculdade de Medicina Veterinária e Zootecnia da Universidade de São Paulo.

STAMP, J.T. Enzootic abortion in ewes. I. Transmission of the disease. Veterinary Record, v.62, p.251-254, 1950.

SZEREDI, L.; BACSADI, A. Detection of Chlamydophila (Chlamydia) abortus and Toxoplasma gondii in smears from cases of ovine and caprine abortion by the streptavidin-biotin method. Journal of Comparative Pathology, v.127, n.4, p.257-63, 2002.

STORZ, J. et al. The isolation of a viral agent from epizootic bovine abortion. Journal of the American Veterinary Medical Association, v.137, p.509-514, 1960.

STORZ, J. et al. Isolation of a psittacosis agent (Chlamydia)from semen and epididymis of bulls with seminal vesiculitis syndrome. American Journal of Veterinary Research, v.29, p.549-555, 1968.

TAKAHASHI, T. et al. Phylogenetic analysis of Chlamydia psittaci from birds based on 16S rDNA gene sequences. Journal of Clinical Microbiology, v.35, p.2908-2914, 1997.

THOMA, R. et al. Chlamydiae in porcine abortion. Veterinary Pathology, v.34, p.467-469, 1997.

VANROMPAY, D. et al. Chlamydia psittaci infections: a review with emphasis on avian chlamydiosis. Veterinary Microbiology, v.45, p.93-119, 1995.

WATSON, M.W. et al. Genetic diversity and identification of human infection by amplification of chlamydial 60-kilodalton cysteine-rich outer membrane protein gene. Journal of Clinical Microbiology, v.29, p.1188-1193, 1991.

WITTENBRINK, M.M. et al. Infection of the bovine female genital tract with Chlamydia psittaci as a possible cause of infertility. Repreoduction of Domestic Animals, v.28, p.129-136, 1993a.

WITTENBRINK, M.M. et al. Endometrits in cattle experimentally induced by Chlamydia psittaci. Journal of Veterinary Medicine, Series B, v.40, p.437-450, 1993 b. 